\title{
The differential effects of emotional salience on direct associative and relational memory during a nap
}

\author{
Sara E. Alger ${ }^{1,2}$ • Jessica D. Payne ${ }^{1}$
}

Published online: 26 September 2016

(C) Psychonomic Society, Inc. 2016

\begin{abstract}
Relational memories are formed from shared components between directly learned memory associations, flexibly linking learned information to better inform future judgments. Sleep has been found to facilitate both direct associative and relational memories. However, the impact of incorporating emotionally salient information into learned material and the interaction of emotional salience and sleep in facilitating both types of memory is unknown. Participants encoded two sets of picture pairs, with either emotionally negative or neutral objects paired with neutral faces. The same objects were present in both sets, paired with two different faces across the sets. Baseline memory for these directly paired associates was tested immediately after encoding, followed by either a $90-$ min nap opportunity or wakefulness. Five hours after learning, a surprise test assessed relational memory, the indirect association between two faces paired with the same object during encoding, followed by a retest of direct associative memory. Overall, negative information was remembered better than neutral for directly learned pairs. A nap facilitated both preservation of direct associative memories and formation of relational memories, compared to remaining awake. Interestingly, however, this sleep benefit was observed specifically for neutral directly paired associates, while both neutral
\end{abstract}

Sara E. Alger

salger@nd.edu

1 Department of Psychology, University of Notre Dame, 118 Haggar Hall, Notre Dame, IN 46556, USA

2208 Haggar Hall, Notre Dame, IN 46556, USA and negative relational associations benefitted from a nap. Finally, REM sleep played opposing roles in neutral direct and relational associative memory formation, with more REM sleep leading to forgetting of direct associations but promoting relational associations, suggesting that, while not benefitting memory consolidation for directly learned details, REM sleep may foster the memory reorganization needed for relational memory.

Keywords Emotion · Associative memory · Naps · Sleep · Inference $\cdot$ Relational memory

In our encounters with the everyday stream of information, we do not encode and store all individual experiences in a veridical fashion. Rather, new information is integrated with old, and overlapping details are consolidated into schemata, or networks linked by common features of related memories, to flexibly form novel relationships (Eichenbaum, 2004; Eichenbaum \& Cohen, 2001). We use these new representations derived from related memories to adaptively make inferential judgments about future experiences. However, our everyday experiences are not devoid of emotion. Emotional salience, defined by the valence (negative to positive) and arousal (calming to arousing) of an experience, is a biologically adaptive cue that can influence how an event is remembered and possibly how it is integrated in memory. In addition to the influence of emotion, sleep during the consolidation and integration period of a new memory may impact its fate. This experiment investigates the separate and interacting contributions of emotional salience and sleep to the consolidation of direct associative and relational memories, the indirect associations between two memories that share overlapping components. 


\section{Direct associative memories and relational memory formation}

First, however, let us provide an example of how relational associations arise from overlapping, directly learned experiences. Imagine you are studying the United States' highway system. You learn that you can travel south from Chicago to St. Louis on Interstate 55 and that you can also travel south on I-55 from St. Louis to Memphis. You are now able to interrelate these two directly learned facts and infer that you can use I55 to travel from Chicago to Memphis, despite never having directly learned this information. In an experimental learning session, two directly paired items (AB), typically word or picture pairs, are experienced together, and the hippocampus works to link the internal representations of $\mathrm{A}$ and $\mathrm{B}$ to form an associative bond (Bunsey \& Eichenbaum, 1996; Davachi \& Wagner, 2002). This direct associative memory is subsequently replayed (Staresina, Alink, Kriegeskorte, \& Henson, 2013), optimally during offline periods of rest (Schlichting \& Preston, 2014) or sleep (Wilson \& McNaughton, 1994). This replay involves reactivation in hippocampal-neocortical (e.g., ventromedial prefrontal cortex; vmPFC) circuits, which strengthens the relationship between A and B through synaptic plasticity (Sutherland \& McNaughton, 2000; Wilson \& McNaughton, 1994), and leads to superior subsequent memory for the pair (e.g., Tambini, Ketz, \& Davachi, 2010). However, when a new pair is then introduced that contains an element that overlaps with prior learned associates $(\mathrm{B}-\mathrm{C})$, the new and existing memories are in conflict. The conflict arises because A and B are known to belong together as a pair, but now B is also being paired with C. Introduction of this conflicting information is thought to reactivate the previously learned pair (A-B) as well. Resolution of this conflict has been shown to involve an increase in connectivity between the hippocampus and the vmPFC (van Kesteren, Fernández, Norris, \& Hermans, 2010; Zeithamova, Dominick, \& Preston, 2012), with activity in the vmPFC phase locked to hippocampal theta oscillations that are prominent both in waking activity and during rapid eye movement (REM) sleep (Sirota \& Buzsáki, 2005). Furthermore, the degree of activation of the vmPFC and hippocampus during this conflict predicts the successful formation of indirect, relational associations $(\mathrm{A}-\mathrm{C})$, which resolves the conflict by incorporating how $\mathrm{B}$ is related to $\mathrm{A}$ and $\mathrm{C}$ and defining the relationship between all three components (van Kesteren et al., 2010; Zeithamova et al., 2012). This connectivity is thought to foster creation of a new schema, or modification of the existing schema, to now include the direct relationships of $\mathrm{A}$ to $\mathrm{B}$ and $\mathrm{B}$ to $\mathrm{C}$, as well as the indirectly linked relational pair, $\mathrm{A}$ and $\mathrm{C}$ (Preston \& Eichenbaum, 2013).

\section{The role of emotion in direct associative and relational memory formation}

Emotionally salient information is typically better remembered than neutral, being more useful to retain to inform future decisions (e.g., Kensinger, 2009; Mather \& Sutherland, 2011). We anticipated one of two possible outcomes to pairing negative and neutral stimuli together in our study, using an A-B, $\mathrm{B}-\mathrm{C}$ design, as described above. Previous evidence from our lab examining memory for complex emotional stimuli has found that, even within stimuli, memory for emotionally salient components is enhanced, but at the expense of memory for the neutral contextual details, an effect known as the emotional memory trade-off effect (Kensinger, Garoff-Eaton, \& Schacter, 2006; Kensinger, 2009; Payne \& Kensinger, 2010; Payne et al., 2015; Payne, Stickgold, Swanberg, \& Kensinger, 2008). A real-life example of this effect is the weapon-focus effect (Loftus, Loftus, \& Messo, 1987), in which the victims of a robbery report superior memory for the details of the weapon that had been present, but at the expense for memory for the face of the assailant.

Just like the emotional memory trade-off effect, the emotionally salient component of the pair in the current study could potentially narrow the focus of attention to the emotional half of the pair, thus impeding the formation of the association between items. Such results were reported by Bisby and Burgess (2014), who demonstrated that negative affect impaired the formation of negative-neutral directly learned image associations, compared to neutral only pairs, but increased memory for the negative image alone when memory for single items was tested. According to this idea, in the current study, in which we asked participants to pair emotionally negative or neutral objects with neutral faces, we would expect to see impaired memory for negative face-object pairs (A-B, B-C) compared to neutral pairs. This would, perhaps, be due to a weapon-focus-like effect in which attention would be focused only on the negative object at the expense of linking the neutral face with that object. This impairment would also be reflected in the formation of relational memories (A-C), which are dependent on good encoding of the direct associates, showing weaker indirect associations between faces paired through negative objects.

However, there is a major difference between the emotional memory trade-off task on which this outcome is based and the task used in the current study. In the trade-off studies, the stimuli were incidentally encoded, with attention naturally focusing on the central emotional object as opposed to the neutral context. In the present study, participants were asked 
to actively attend to and associate the two components of the pair to form a representation that encompassed both. An alternative outcome then is that emotion could facilitate the integration of the emotional and neutral items in a pair into a single representation. Thus, the salient nature of the stimuli would result in prioritized binding of peripheral details, making the negative object and neutral face pair a memorable whole (Hadley \& MacKay, 2006; MacKay \& Ahmetzanov, 2005; MacKay et al., 2004; Nummenmaa, Hyönä, \& Calvo, 2006; Phelps, Ling, \& Carrasco, 2006). This facilitation, too, would be reflected in the formation of relational memory, with the better-learned directly associated negative pairs resulting in stronger indirect relational connections between the associated faces (Lau, Tucker, \& Fishbein, 2010).

\section{A Role for Sleep in Direct Associative and Relational Memory Formation}

In addition to emotional salience, we also investigated the impact of sleep on the consolidation of these direct associative and relational memories. Previous research using neutral stimuli only has shown that although it is possible to form direct and relational associations during a waking experience (Preston, Shrager, Dudukovic, \& Gabrieli, 2004; Tambini et al., 2010), sleep facilitates this process. Sleep more efficiently consolidates memory for the explicitly learned relationships, integrating the newly learned information with the old to aid in the creation of these schemata, and strengthening the indirect relational bonds (Ellenbogen, $\mathrm{Hu}$, Payne, Titone, \& Walker, 2007; Lau, Alger, \& Fishbein, 2011; Lau et al., 2010). During sleep, reactivation of hippocampal dependent memories strengthens memories both at the synaptic and systems level (e.g., Buzsáki, 1989; Pavlides \& Winson, 1989; Wilson \& McNaughton, 1994). Through this mechanism, hippocampal-dependent memories, such as the directly learned paired associates and relational memories, are thought to benefit more from sleep than from wakefulness. Slow wave sleep (SWS) is thought to consolidate hippocampal-dependent memories (Gais \& Born, 2004; Plihal \& Born, 1997), and, as mentioned, the hippocampus is crucially involved in not only binding the cortical representations of the directly learned associates but also in relating disparate memory components to foster relational memories (Bunsey \& Eichenbaum, 1996; Dusek \& Eichenbaum, 1997; Preston et al., 2004, Zeithamova et al., 2012). Correspondingly, Lau et al. (2010) found that SWS was a predictor of relational memory performance. However, REM sleep is thought to facilitate integration of new information into existing stores (Stickgold, Whidbee, Schirmer, Patel, \& Hobson, 2000; but see Tamminen, Payne, Stickgold, Wamsley, \& Gaskell, 2010), creative associations (Cai, Mednick, Harrison, Kanady, \& Mednick, 2009), problem solving (Walker, Liston, Hobson,
\& Stickgold, 2002) and other forms of memory transformation, being a state favorable for associative learning (Hasselmo \& Bower, 1993; Landmann et al., 2015). Because creating relational associations involves integrating overlapping new and old pairs and extracting their shared relationship, REM sleep could foster the type of intracortical communication needed for this schematic reorganization (Cai et al., 2009; Durrant, Cairney, McDermott, \& Lewis, 2015). The current study aimed, in part, to investigate the role of sleep in both negative and neutral direct associative and relational memory formation to provide further evidence and clarify the sleep physiology involved (e.g., SWS or REM sleep).

\section{Sleep tends to preferentially benefit emotional memories}

Given that we varied the emotionality of stimuli in this experiment, we consider literature regarding the role of sleep in preferentially consolidating emotional memory, as no previous study has examined the interaction of sleep and emotion specifically in relational memory formation. Sleep has been repeatedly shown to preferentially benefit emotional memories over neutral ones (Hu, Stylos-Allan, \& Walker, 2006; Nishida, Pearsall, Buckner, \& Walker, 2009; Payne \& Kensinger, 2010; Wagner, Gais, \& Born, 2001), although evidence of this effect is not conclusive (Baran, Pace-Schott, Ericson, \& Spencer, 2012), as well as to selectively benefit the emotional components of a memory over the neutral contextual details (Cunningham et al., 2014; Payne, Chambers, \& Kensinger, 2012; Payne et al., 2015; Payne et al., 2008). REM sleep, specifically oscillations in the theta frequency range, has been most often linked to emotional memory consolidation (Nishida et al., 2009). In our study, we anticipated that sleep would facilitate the consolidation of the negative paired associates and the formation of negative relational memories over neutral pairs. We hypothesized that the emotional response to the negative pairs may "tag" those integrated associations for preferential processing during sleep (see Bennion, Mickley Steinmetz, Kensinger, \& Payne, 2015; Cunningham et al., 2014).

We employed a daytime napping design to investigate the interaction of emotional salience and sleep on both direct associative and relational memory performance, using a novel incorporation of negatively salient items. Specifically, this study examined how sleep facilitates the formation of indirect relationships stemming from overlap between learned information, and how the valence of the objects paired with neutral faces affects these associations. We hypothesized that the face-object and face-face pairs that contained or were indirectly associated through negatively salient objects (i.e., A-B, with $\mathrm{B}$ being a negative object, or $\mathrm{A}-\mathrm{C}$, with the same negative object $\mathrm{B}$ in common; see Fig. 1) would be better 


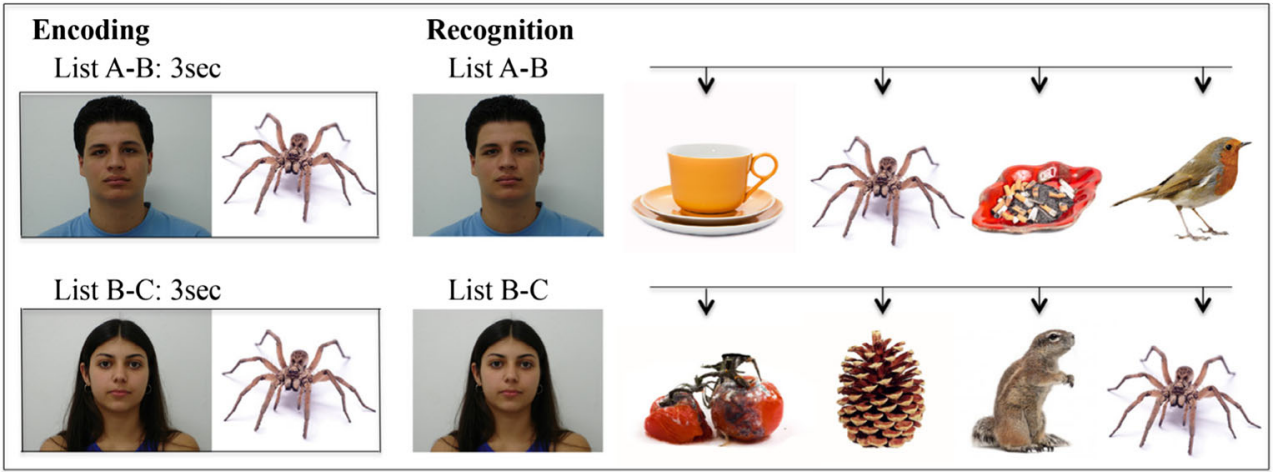

Fig. 1 Procedure for encoding and baseline testing. Participants encoded two sets of face-object pairs, A-B and B-C, with each pair presented for 3 seconds. A forced-choice recognition test to measure baseline memory for these directly associated pairs occurred after each set. Objects were the same between the sets and therefore were paired with two separate faces (A and C), which were then indirectly related through the common object. Half of the objects were negatively salient and half were neutral

exchange for course credit or monetary payment, as well as through flyers posted around campus. This study was conducted according to the principles expressed in the University of Notre Dame Human Subjects Institutional Review Board, with all subjects providing written informed consent.

Before arriving at the lab, participants were contacted via email to ensure eligibility by administering the screening form. They kept a 3-day sleep log to track their sleep-wake times, napping habits, and alcohol and caffeine intake prior to the experimental day. Participants were instructed to get no less than 6 hours of sleep the night before the experimental day and refrain from caffeine, alcohol, nicotine, and unnecessary medication 24 hours before and for the duration of the study. Upon arrival to the lab, participants were asked to sign a consent form explaining the nature of the research and completed a demographics questionnaire as well as several other questionnaires to assess sleep habits and rule out psychopathology; these included the Pittsburg Sleep Quality Index (PSQI; Buysse, Reynolds, Monk, Berman, \& Kupfer, 1989) to assess general sleep quality, the Morningness-Eveningness Questionnaire (MEQ; Horne \& Ostberg, 1976) to assess morningness-eveningness tendencies, the Beck Anxiety Inventory (BAI; Beck, Epstein, Brown, \& Steer, 1988) and State-Trait Anxiety Inventory (STAI, Spielberger, 2010) to assess level of state and trait anxiety, the Beck Depression Inventory-II (BDI; Beck, Ward, \& Mendelson, 1961) to assess depressive symptoms, the Positive and Negative Affect Schedule (PANAS; Watson, Clark, \& Tellegen, 1988) to assess positive and negative affect, and the Stanford Sleepiness Scale (SSS; Hoddes, Zarcone, Smythe, Phillips, \& Dement, 1973) to assess state sleepiness. These measures were used primarily to compare nap and wake groups to ensure they were similar to one another as well as to determine if any participant should be excluded because of scoring in elevated (i.e., clinical) ranges for depression or anxiety, which could impact emotional memory formation. 


\section{Materials}

The stimulus materials consisted of color photographs of 80 different faces on light gray backgrounds and 40 objects of roughly the same size on white backgrounds. Faces were frontal facing and prealigned images from a face database maintained by the Department of Electrical Engineering of FEI, São Paolo, Brazil (Thomaz \& Giraldi, 2010). The set of faces contained an equal number of male and female pictures. Only the pictures that were categorized as having completely neutral facial expressions, facing upright and straight toward the camera, were used.

A pilot study ( $n=20)$ was conducted to justify designations of objects either as neutral or negatively salient. The $40 \mathrm{ob}-$ jects used in the experiment were rated on 1 to 7 scales measuring valence ( 1 as the most negative, 7 as the most positive) and arousal ( 1 as not at all arousing, 7 as highly arousing). Half of the objects were classified as negative based on a low valence score and a higher arousal score $(M \pm S D$; valence $=$ $1.82 \pm 0.35$, arousal $=5.11 \pm 0.53)$. The other half of the objects were classified as neutral based on a moderate valence score and a lower arousal score (valence $=4.44 \pm 0.30$, arousal $=2.68 \pm 0.71)$.

\section{Task}

We used an adapted version of an associative inference task (Bunsey \& Eichenbaum, 1996; Preston et al. 2004; Lau et al., 2010), which was composed of two sets of color photograph pairs, each of a face and an easily identified object. There were 40 photograph pairs in each set, with the same objects in both sets. As mentioned, this study included a novel incorporation of emotionally salient components to examine the impact on both direct paired associates and relational pairs. Participants learned two sets of 40 direct face-object paired associates (a set of A-B pairs and a set of B-C pairs). Either negative (e.g., a bloody knife) or neutral (e.g., a teacup) objects (B items; 20 of each valence) were common elements shared by two sets of neutral faces ( $\mathrm{A}$ and $\mathrm{C}$ items). Four lists consisting of the two sets of A-B and B-C pairs were created to balance presentation and avoid list and order effects. Between Lists 1 and 2, the pairs in the A-B and B-C sets were viewed in the same sequence, but List 1 presented $\mathrm{A}-\mathrm{B}$ pairs first, followed by $\mathrm{B}-\mathrm{C}$ pairs, whereas List 2 did this in reverse. Lists 3 and 4 contained the same faces and objects in each set as in the first two lists, but in a different sequence. List 3 presented $\mathrm{A}-\mathrm{B}$ pairs first, followed by B-C pairs; List 4 did this in reverse. Additionally, in each set, half of the objects were paired with male faces and half were paired with female faces to eliminate sex bias. All faces across the four counterbalanced lists appeared with both a negative and a neutral object. Each object appeared with both a male and female face at some point across the lists.
Participants had one exposure to each of the A-B and B-C picture sets. This encoding protocol was found to be optimal based on a second pilot study $(n=20)$ that yielded a level of retention of the face-object pairs high enough to ensure adequate baseline retention of direct associative memories (approximately $75 \%$ retention when tested immediate after encoding).

\section{Procedures}

At approximately 12 p.m., the participants arrived in the Sleep, Stress, and Memory Lab, signed informed consent, and completed a set of questionnaires [PSQI, MEQ, STAIX2 (assessing trait), BAI, STAI-XI (assessing state), and PANAS], which also included the Stanford Sleepiness Scale (SSS). They then encoded all pairs. Each pair was displayed for 3,000 ms with an interstimulus interval of $500 \mathrm{~ms}$. Participants were instructed to pay close attention to each picture pair, encouraged to associate the face and object in a meaningful way, and told they would be tested for retention of the face-object pairs. Baseline tests were administered after the completion of each set presentation (see Fig. 1).

The baseline testing session that immediately followed each encoded set (i.e., one presentation of $\mathrm{A}-\mathrm{B}$ followed by the $\mathrm{A}-\mathrm{B}$ pairs baseline test, then one presentation of $\mathrm{B}-\mathrm{C}$ followed by $\mathrm{B}-\mathrm{C}$ pairs baseline test) consisted of a forcedchoice recognition test of direct associative memory. In this test, a cued face was displayed with a set of four objects (two negative, two neutral), one previously paired with the cued face and three other choices. Similar to the testing procedure used by Preston et al. (2004) and Lau et al. (2010), all incorrect choices, or foils, were stimuli that had previously been paired with other faces so that individual stimuli were familiar, controlling for the possibility that choices could be eliminated because they were recognized as novel. Additionally, all four object choices in any given question were ensured to all have been previously paired with a face of the same sex. Memory for all encoded pairs was tested. Participants were told at the end of the first session that they would be tested in this manner once more during the second session, but were only aware of this direct associates test.

Test subjects were separated into wake $(n=29)$ and nap ( $n$ $=29$ ) groups, the former of which were free to leave the lab until the final session and the latter of which were prepared for polysomnography. At around 1:00 p.m., nap subjects were given a 90-minute sleep opportunity lasting until about 2:30 p.m. Nap participants were monitored online using digital EEG acquisition software (Comet System-Grass/Twin PSG Clinical Software) using a standard polysomnographic montage, which included electroencephalography (EEG; F3, F4, C3, C4, Cz), electrooculography (EOG), and chin electromyography (EMG) channels, each referenced to contralateral mastoids. If participants obtained SWS or REM sleep during 
this time, they were allowed to sleep until they transitioned out of these stages, to reduce sleep inertia and disorientation. After awakening, electrodes were removed and nap participants were allowed to leave the lab until the second session, approximately 2.5 hours later. We chose to have this delay after the nap before the retest session so that the nap was a smaller portion of the retention period, with waking activity outside of the lab occurring in both groups to better equate their experiences. This helps to address potential criticism that any benefits in the nap group are due to passive interference, such that the nap group performs better simply because they were protected from external stimuli during the whole of the retention period. This design also gives the nap group ample time to recover from any sleep inertia experienced after awakening.

At 5 p.m., all participants returned to the lab and completed a second set of questionnaires similar to the previous session (BDI, STAI-XI, PANAS, and SSS). They were first unexpectedly (as confirmed by exit questioning at debriefing) tested on their memory for the 40 indirect relational pairs formed by two faces sharing a relationship with the same object across the A$\mathrm{B}$ and $\mathrm{B}-\mathrm{C}$ lists. In this test, participants were presented with a face and asked to choose, among four other faces, the one that was paired with the same object as the cued face (see Fig. 2). This relationship was not explicitly stated by any previous instructions, but was a measure of associative inference. Each face seen during the learning session was either presented as the cued face or the correct choice in the four potential choices; half of the cued faces were from the list learned first (A from A-B) and half were from the second list (C from BC), resulting in 40 forced-choice questions. This surprise test was then followed by a final retest, which participants expected, on all 80 previously learned direct paired associates (A-B and $\mathrm{B}-\mathrm{C}$, together), in randomized order to eliminate the list order effect, similar to baseline testing. The surprise relational memory test always came before the retesting of direct pairedassociates memory. This was done to avoid a priming effect and because participants could conceivably form additional relational associations during the final test of direct associates that had not been formed during the initial encoding session or consolidation period.

To address our hypotheses, we compared groups on the change in performance from baseline to retest for the emotional and neutral direct paired-associates performance using a mixed ANOVA. Relational memory performance was first adjusted by partialling out the influence of the covariates of baseline performance for direct associates, as formation of relational memory is highly dependent on how well the directly learned pairs are encoded (Lau et al., 2010). We then performed a mixed ANOVA to examine group differences in emotional and neutral relational memory. The relationship between sleep, particularly SWS and REM sleep, and both direct associative and relational memory performance was assessed with Pearson's correlation.

\section{Results}

Baseline performance was assessed by calculating the proportion of correctly remembered face-object direct associate pairs (number of correct pairs/total possible number of pairs) for both the $\mathrm{A}-\mathrm{B}$ and $\mathrm{B}-\mathrm{C}$ sets for each valence (i.e., $\mathrm{A}-\mathrm{B}$ negative and neutral, $\mathrm{B}-\mathrm{C}$ negative and neutral). No significant difference in baseline performance was found between $\mathrm{A}-\mathrm{B}$ $(M \pm S E M, 0.72 \pm 0.02)$ and $\mathrm{B}-\mathrm{C}(0.74 \pm 0.02)$ sets across valences, $F(1,56)=2.14, p=.15$, or as an interaction between set and valence (A-B negative $0.74 \pm 0.02$, A-B neutral 0.70 \pm 0.02 , B-C negative $0.75 \pm 0.02$, B-C neutral $0.74 \pm 0.03$ ), $F(1,56)=1.77, p=.19$. Therefore, for all further analyses, performance was calculated for negative and for neutral paired associates collapsed across lists (i.e., baseline negative, baseline neutral). Performance for direct paired associate memory was assessed similarly at retest. Additionally, relational memory was calculated by dividing the number of correctly identified face-face pairs by the total possible number of pairs for both negative and neutral related pairs (i.e., faces that were associated through negative or neutral items, respectively).

\section{Sleepiness and Questionnaire Measures}

Independent-samples $t$ tests were used to verify that there were no differences between groups on any questionnaire, sleepiness scale, or sleep log data, such as amount of sleep achieved the night before the experimental day (see Table 1). There were no significant differences between groups on any measure, with the exception of the Stanford Sleepiness Scale (SSS) in the second session. As might be expected, wake participants had a significantly higher SSS score (meaning they were more sleepy) in their second session $(M=2.69 \pm$ $0.17)$ than did those in the nap group $(M=1.93 \pm 0.16), t(56)=$ 3.33, $p=.002$. To determine whether sleepiness impacted performance in the wake condition, correlations between the second session SSS scores and all performance measures during retest as well as change between baseline and retest were conducted, with no significant relationships found; negative relational memory, $r=.03, p=.81$; neutral relational memory, $r=.05, p=.74$; negative direct associative memory, $r=.18, p$ $=.19$; neutral direct associative memory, $r=.12, p=.36$; change in negative direct associative memory, $r=-.004, p=$ .98; change in neutral direct associative memory, $r=-.05, p=$ .81 . These findings indicate that greater sleepiness in the wake group is not responsible for their poorer performance.

\section{Sleep data}

The sleep recordings were scored using the standard criteria of Rechtschaffen and Kales (1968). The nap group slept on average in minutes $58.38 \pm 4.92(M \pm S E M)$ with an average sleep onset latency of $10.20 \pm 1.96$ and REM sleep latency of 


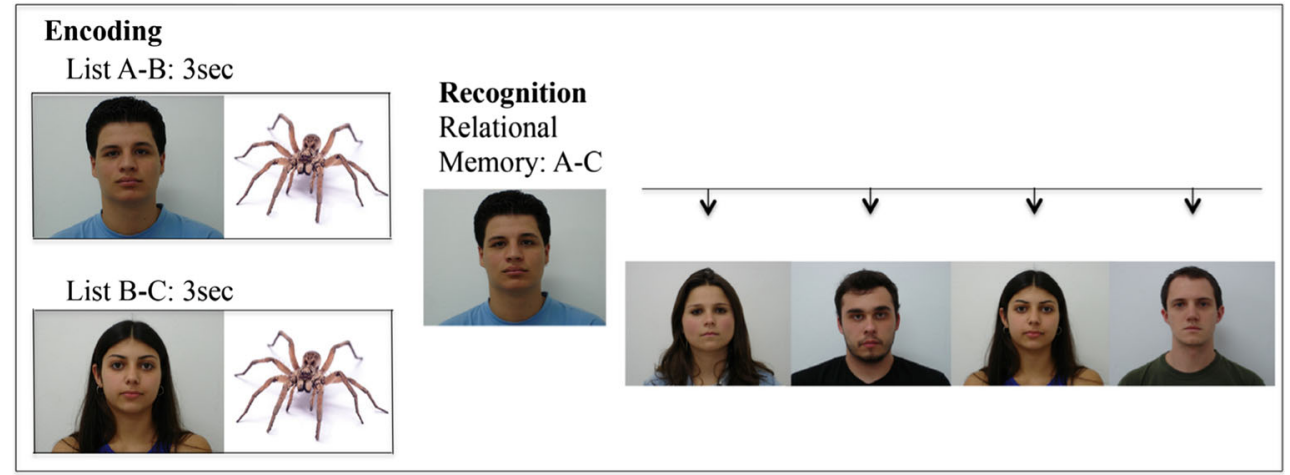

Fig. 2 Relational memory testing. The surprise relational memory test was given after the nap/wake retention interval. We tested indirect memory for the two faces related through the common object paired with each during the encoding session. During the relational memory recognition

$62.03 \pm 3.30$. On average in minutes, nap subjects obtained $11.77 \pm 1.34$ of Stage 1 sleep, $26.93 \pm 2.37$ of Stage 2 sleep,

Table 1 Comparison of demographic and questionnaire measures between conditions

Condition

\begin{tabular}{|c|c|c|c|c|c|c|}
\hline \multirow[b]{2}{*}{ Measurement } & \multicolumn{2}{|l|}{ Nap } & \multicolumn{2}{|l|}{ Wake } & \multicolumn{2}{|c|}{ Difference } \\
\hline & Mean & SEM & Mean & SEM & $t$ & $p$ \\
\hline Age (years) & 19.79 & 0.23 & 19.48 & 0.18 & -1.06 & 0.30 \\
\hline Sleep prior night (hours) & 7.02 & 0.16 & 6.69 & 0.21 & -1.25 & 0.22 \\
\hline MEQ & 45.79 & 2.04 & 42.93 & 1.39 & -1.16 & 0.25 \\
\hline PSQI & 5.24 & 0.43 & 4.55 & 0.37 & -1.21 & 0.23 \\
\hline STAI X-2 & 43.41 & 1.60 & 43.59 & 1.24 & 0.09 & 0.93 \\
\hline BDI & 6.21 & 0.96 & 7.86 & 1.00 & 1.20 & 0.24 \\
\hline BAI & 8.03 & 0.94 & 9.55 & 1.00 & 1.10 & 0.27 \\
\hline \multicolumn{7}{|l|}{ Session 1} \\
\hline PANAS (positive affect) & 23.31 & 0.79 & 23.76 & 0.95 & 0.36 & 0.72 \\
\hline PANAS (negative affect) & 14.52 & 1.00 & 15.69 & 0.96 & 0.85 & 0.40 \\
\hline STAI X1 & 37.72 & 1.77 & 39.28 & 1.68 & 0.64 & 0.53 \\
\hline SSS & 2.83 & 0.17 & 2.79 & 0.20 & -0.13 & 0.90 \\
\hline \multicolumn{7}{|l|}{ Session 2} \\
\hline PANAS (positive affect) & 23.10 & 1.13 & 22.21 & 1.05 & -0.58 & 0.56 \\
\hline PANAS (negative affect) & 13.76 & 0.58 & 14.93 & 0.71 & 1.42 & 0.15 \\
\hline STAI X2 & 36.62 & 1.57 & 39.21 & 1.33 & 1.66 & 0.17 \\
\hline SSS & 1.93 & 0.16 & 2.69 & 0.17 & 3.33 & 0.00 \\
\hline
\end{tabular}

Note. The nap group and wake group were compared across all measures collected from demographics, questionnaires, and sleep logs. No significant differences were found between the groups on any measure except for the Stanford Sleepiness Scale (SSS) in Session $2(p=.002)$. SSS scores in Session 2 were not correlated with any measure of performance. Questionnaires used were the Morningness Eveningness Questionnaire (MEQ), Pittsburg Sleep Quality Index (PSQI), State-Trait Anxiety Inventory (STAI-X1 and X2), Beck Depression Inventory-II (BDI), Beck Anxiety Inventory (BAI), Positive and Negative Affect Schedule (PANAS), and Stanford Sleepiness Scale (SSS) test, participants were presented with one face from either the A-B or the B-C set and asked to choose among four faces which one had been paired with the same object

$13.55 \pm 2.85$ of SWS, and $6.13 \pm 1.50$ of REM sleep. In relation to total sleep time, the nap participants had $24.21 \pm$ $3.22 \%$ of Stage 1 sleep, $48.82 \pm 3.03 \%$ of Stage 2 sleep, $18.50 \pm 3.73 \%$ of SWS, and $8.00 \pm 1.90 \%$ of REM sleep.

\section{Direct paired associate (face-object) memory}

To verify that there were no significant differences between groups for negative or neutral direct paired associate memory at baseline, independent-samples $t$ tests were conducted. No significant differences were found for any measure, negative: $M \pm S E M$ for nap $0.73 \pm 0.03$, wake $0.76 \pm 0.03, t(56)=0.93$, $p=.35$; neutral: nap $0.70 \pm 0.03$, wake $0.75 \pm 0.03, t(56)=$ $1.18, p=.24$, suggesting that all groups encoded the material equally (see Table 2). A $2 \times 2 \times 2$ mixed ANOVA was conducted to examine retention of face-object pairs across the intervening period containing either sleep or wake, with condition (nap, wake) as the between-subjects factor and session (baseline, retest) and valence (negative, neutral) as the withinsubjects factors. This analysis revealed a significant main effect of session, $F(1,56)=261.50, p<.001, \eta_{\mathrm{p}}{ }^{2}=0.82$, indicating that all subjects' performance declined from baseline to retest, and a significant main effect of valence, $F(1,56)=7.08$, $p=.01, \eta_{\mathrm{p}}{ }^{2}=0.10$, indicating that faces paired with negative objects were remembered better compared to neutral faceobject pairs, overall. There was also a significant two-way interaction between session and group, $F(1,56)=6.79, p=$ $.01, \eta_{\mathrm{p}}{ }^{2}=0.12$. As hypothesized, nap subjects retained memory for significantly more direct-associative pairs over time than did wake subjects. This finding was further qualified by a significant three-way interaction between group, session, and valence, $F(1,56)=4.46, p=.04, \eta_{\mathrm{p}}^{2}=0.07$. Post hoc analyses revealed that when examining the change in memory performance between baseline and retest for the negative faceobject pairs, there was no difference between the wake and nap conditions, $t(56)=-.84, p=.41$. However, napping specifically facilitated the preservation of neutral directly paired 
Table 2 Performance for direct associative and relational memory

\begin{tabular}{|c|c|c|c|c|c|c|c|c|}
\hline \multirow[t]{2}{*}{ Condition } & \multicolumn{2}{|c|}{ Baseline (B) } & \multicolumn{2}{|c|}{ Retest (R) } & \multicolumn{2}{|c|}{ Change (R-B) } & \multicolumn{2}{|c|}{ Relational Memory } \\
\hline & Mean & SEM & Mean & SEM & Mean & SEM & Mean & SEM \\
\hline \multicolumn{9}{|c|}{ Neutral performance } \\
\hline Nap & 0.70 & 0.03 & 0.58 & 0.03 & $-0.12 * *$ & 0.02 & $0.44 * *$ & 0.02 \\
\hline Wake & 0.75 & 0.03 & 0.54 & 0.03 & -0.21 & 0.02 & 0.34 & 0.03 \\
\hline \multicolumn{9}{|c|}{ Negative performance } \\
\hline Nap & 0.73 & 0.03 & 0.57 & 0.02 & -0.15 & 0.01 & $0.45 *$ & 0.02 \\
\hline Wake & 0.76 & 0.03 & 0.59 & 0.03 & -0.17 & 0.02 & 0.37 & 0.03 \\
\hline
\end{tabular}

Note. Performance was measured as a proportion of total possible correct pairs in the direct associative memory forced-choice task during the baseline test and retest. The change in performance over time (retest minus baseline performance) was significantly different between nap and wake conditions for total performance, regardless of valence, but specifically for the neutral face-object pairs. Likewise, performance on the relational memory test, measured as a proportion of correctly associated face-face pairs, was also significantly different between groups, with baseline performance as a covariate, for total and neutral relational face- face pairs. Bolded values indicate significant $t$-test comparisons.

$* p<.05 . * *<.01$

associates over time, compared to the wake condition, $t(56)=$ $-3.17, p=.002$, CI $[-0.14,-0.03]$ (see Fig. 3).

Taken together, these results support our hypothesis that naps facilitate the preservation of directly paired associate memory over time, compared to an equal amount of time spent awake. However, contrary to our predictions, napping benefitted neutral associative memory but not negative salient pairs, compared to remaining awake.

\section{Relational (face-face) memory}

Unlike direct associative memory, where performance was tracked from baseline to retest, there was no baseline performance to which relational memory could be compared, by nature of the task. Relational memory performance is

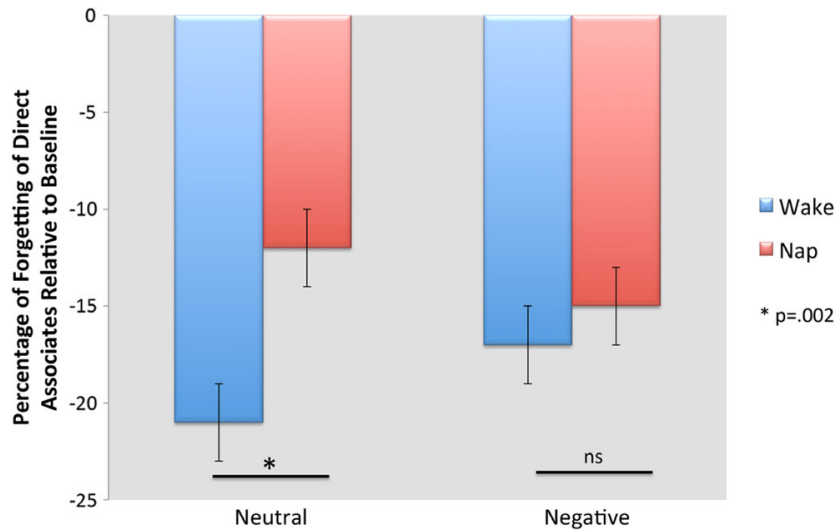

Fig. 3 Forgetting of direct associative memory across time. We compared the percentage of forgetting of direct associative memory over time, calculated by subtracting baseline performance from retest performance for each individual. Presented are the average percentages of forgetting from baseline performance for the nap and wake groups. The nap was found to significantly preserve memory for neutral face-object pairs $(p=.002)$ compared to the waking control condition. No such benefit from a nap was found for negative face-object pairs $(p=.41)$. Error bars represent the standard error of the mean (SEM) dependent on how well the initial directly paired associates were learned, enabling the relational connections between pairs to form (Lau et al., 2010). In our data, baseline memory for neutral direct associates was significantly positively correlated with neutral relational memory $(r=.67, p<.001)$, as were negative direct associates baseline and negative relational memory $(r=.81, p<.001)$. To account for between-group memory variability between nap and wake participants, we used relevant baseline performance of the face-object pairs as a covariate (e.g., negative face-object pairs at baseline as a covariate for face-face pairs related through negative objects), similar to Lau et al. (2010). We first partialled out these variables from the relational memory data (e.g., adjusting neutral relational memory performance to account for the influence of baseline neutral direct associate memory performance) before conducting an ANOVA. Performance at retest was not used as a covariate, as we expected that probing memory for the A - C (face-face) pairs would have a priming effect on the subsequent direct associates retest. Using a 2 (condition) $\times 2$ (valence) mixed ANOVA, we found a main effect of condition, $F=10.95, p=.002, \eta_{\mathrm{p}}{ }^{2}=0.16$, demonstrating superior relational memory formation following a period of sleep compared to a period of wakefulness. We found no effect of valence or interaction of valence and condition, indicating that sleep, compared to wake, was beneficial to face-face memory related both through negative, $t(56)=-2.45, p=.02, \mathrm{CI}$ $[-0.14,-0.01]$, and neutral objects, $\mathrm{t}(56)=-2.92, p=.005$, CI $[-0.16,-0.03]$; see Fig. 4.

To further investigate this sleep effect, we conducted an exploratory analysis of relational memory based on memory for the $\mathrm{A}-\mathrm{B}$ and $\mathrm{B}-\mathrm{C}$ pairs at baseline. We isolated, for each individual, only those responses in the $\mathrm{A}-\mathrm{C}$ test for which the individual had correctly paired the $\mathrm{A}-\mathrm{B}$ and $\mathrm{B}-\mathrm{C}$ items during baseline testing. Our logic was that since relational memory is dependent on accurate direct associate memory, the effect of 


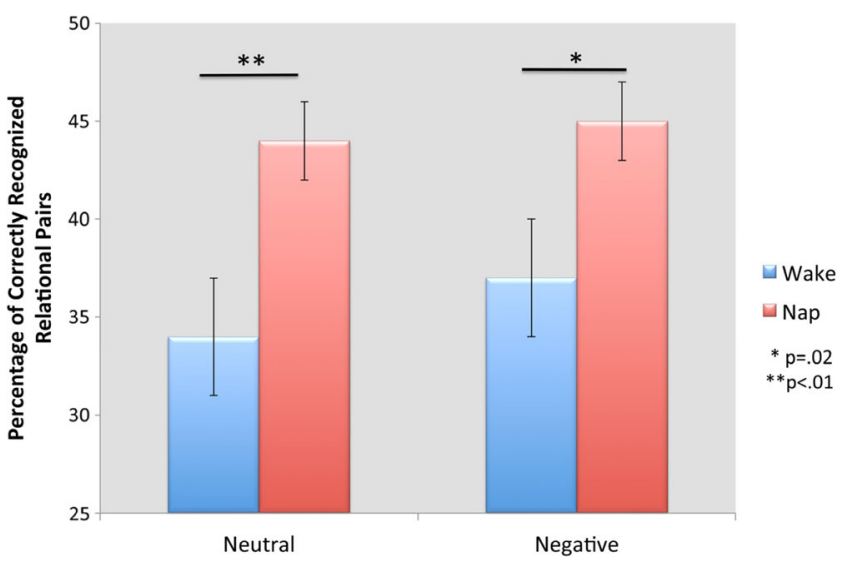

Fig. 4 Relational memory performance. The nap benefitted the formation of relational memories for all the indirectly associated faceface pairs, regardless of valence, with the nap group recognizing significantly more relational associations than the wake group for

sleep on relational memory formation would be clearest in these pairs. We then reran the 2 (condition) $\times 2$ (valence) ANOVA without needing to control for baseline performance now that this was set at $100 \%$ for all A-C pairs examined. The results were similar to the original analysis, with no main effect of valence or interaction between valence and condition, but with a nearly significant, $F(1,56)=3.70, p=.06$, main effect of condition, demonstrating that the nap group recognized more indirectly related pairs overall than the wake group. This was not as strong of an effect as in the original analysis using all pairs; however, in limiting the analysis to only those pairs that were correctly retrieved at baseline, we also eliminated the possibility that sleep could promote gains in memory for both direct and relational associations (i.e., correct memory at retest when incorrect at baseline).

\section{Sleep measures and memory}

Limited correlational analyses were planned and conducted between sleep measures of interest reflected as percentage of total sleep time (SWS\% and REM\%) and changes in performance in direct associate memory between baseline and retest, as well as with relational memory, correcting for multiple comparisons using the Bonferroni correction. One nap subject was excluded from these analyses due to a brief gap in the PSG recording that made it impossible to accurately calculate duration and percentage of total sleep time of any sleep measure. We found that the percentage of REM sleep obtained in the nap correlated with the change in memory for the neutral face-object pairs, indicating that more REM sleep was related to more forgetting of the neutral directly paired associates between baseline and retest sessions ( $r=.47, p=.01$; see Fig. 5). Conversely, the percentage of REM sleep was positively correlated with memory for faces related through neutral neutral $(p=.005)$ and negative $(p=.02)$ information. Performance at baseline is used as a covariate to compare relational memory between conditions and is reflected in the graph as adjusted values. Error bars represent $S E M$

objects ( $r=.49, p=.01$; see Fig. 6). No other correlations emerged between any other stage of sleep and any other measure of behavioral performance. Notably, there were no correlations between performance and total sleep time.

Since roughly half of the nap participants obtained REM sleep, we followed these correlational analyses up by excluding those participants who did not reach REM sleep (the data points on the $y$-axis in Figs. 5 and 6) to see if these sleep and behavioral relationships were maintained. Correlating REM\% with direct associate and relational memory in this subgroup of participants who obtained REM sleep $(n=15)$ yielded significant relationships mirroring the results of the full set of nappers. REM sleep correlated with the change in memory for the neutral face-object pairs, indicating that more REM sleep was related to more forgetting of the neutral directly

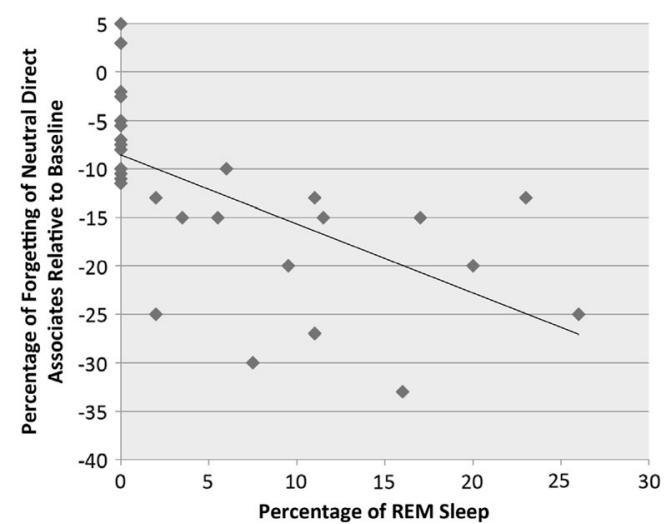

Fig. 5 Correlation between REM sleep and forgetting of neutral direct associates. The percentage of REM sleep obtained in the nap was found to correlate with the change in memory for the neutral face-object pairs, indicating that more REM sleep was related to more forgetting over time of the neutral directly paired associates between baseline and retest sessions $(r=.47, p=.01)$. Negative values on the $y$-axis indicate the decrease in performance relative to baseline measures 


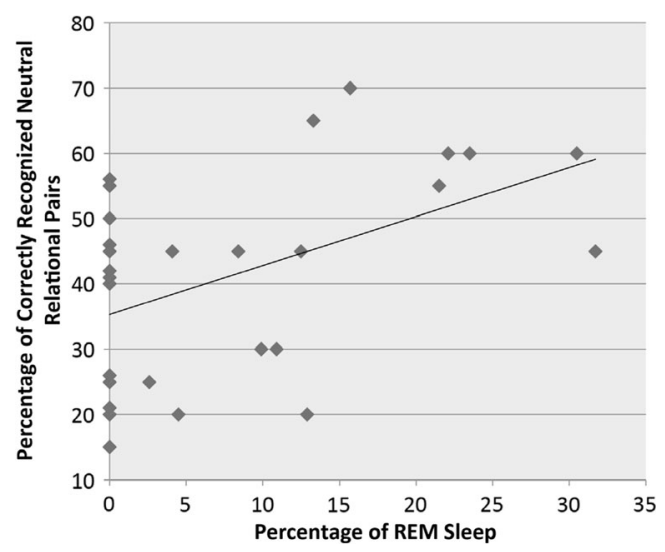

Fig. 6 Correlation between REM sleep and neutral relational memory. Examining performance in the nap condition on the relational memory task for the face-face pairs related through neutral objects, we found a significant correlation between percentage of REM sleep and neutral relational memory $(r=.49, p=.01)$

paired associates over time $(r=.57, p=.03)$. Conversely, the percentage of REM sleep was positively correlated with memory for faces related through neutral objects $(r$ $=.57, p=.03$ ).

\section{Discussion}

In this study, we demonstrated that participants who napped exhibited better retention of direct associative memory (i.e., face-object associations - A-B and B-C pairs) and better relational memory formation (i.e., face-face associations $-\mathrm{A}-\mathrm{C}$ pairs) than their counterparts who remained awake. By using a nap design, we were able to control for time-of-day confounds, with equivalent times of training and testing between groups without the confounds associated with sleep deprivation or comparing overnight sleep to daytime wake. The results here can also not be accounted for by between-group differences in sleepiness, level of depression and anxiety, or morningness or eveningness type. Overall, our results indicate that sleep-specific processes promote these two related forms of declarative memory, likely through reactivation of the direct associative memories and integration of the related components to form relational associations (Gais \& Born, 2004; Wilson \& McNaughton, 1994). This supports what has been found in previous literature (Lau et al., 2010).

However, when we examine the impact of emotional salience on these effects, which was a main focus of the study, we see a more complex picture. Little is known about the influence of emotional salience on memory for directly paired associates, as relatively few studies have paired emotional and neutral components together. To our knowledge, this is the first study exploring the role of sleep using emotionally salient information as a part of the learned pairs to measure the impact on relational memory formation.
We hypothesized that objects with greater salience, such as emotionally negative, highly arousing objects, would facilitate encoding and consolidation of memory for directly paired associates as well as the formation of relational memory, compared to neutral information, in line with the prioritized binding theory (Hadley \& MacKay, 2006; MacKay \& Ahmetzanov, 2005; MacKay et al., 2004). Accordingly, we found a main effect of valence, demonstrating that, across sessions, negative salience led to significantly greater memory for the negative face-object pairs compared to neutral face-object pairs. This did not extend to relational memory, as performance was equal between negative and neutral relational pairs, although performance for relational memory was only tested after the sleep-wake manipulation. Based on the abundant literature citing the benefits of sleep for emotional memory over neutral memory (Hu et al., 2006; Nishida et al., 2009; Payne \& Kensinger, 2010; Wagner et al., 2001), we further hypothesized that this influence of emotional salience would interact with sleep, resulting in greater sleep-related benefits for both the negative direct associate and relational memories. Contrary to our hypothesis, the nap resulted in superior preservation of memory for neutral direct associative memory, compared to the wake group, with memory decaying $12 \%$ compared to $21 \%$, respectively, from baseline to retest, while the wake and nap groups performed similarly for negative direct associates (see Fig. 3). However, our results clearly demonstrate a benefit of sleep for relational memory formation, as in prior studies (Ellenbogen et al., 2007; Lau et al., 2011; Lau et al., 2010), and this benefit was seen for all relational connections, regardless of valence.

When we originally considered predictions about how adding emotional salience to the $\mathrm{A}-\mathrm{B} / \mathrm{B}-\mathrm{C}$ task would impact memory and interact with sleep-based consolidation processes, we first reflected on previous work from our lab demonstrating selective preservation of emotional components of scenes in the emotional memory trade-off task (Payne et al., 2008; Payne \& Kensinger, 2011; Payne et al., 2015). In these experiments, emotional objects are better remembered than neutral objects, but at the expense of memory for neutral information in the background, and sleep magnifies this trade-off effect, boosting memory specifically for the emotional images. Furthermore, there is evidence that shows that emotionally salient information that is attended to and results in an arousal response is marked for preferential consolidation during sleep, also resulting in stronger activation in the hippocampus and amygdala (Bennion et al., 2015; Cunningham et al., 2014). Thus, in this study we considered that the emotional item of a pair might draw focus away from the neutral item, thereby impeding the ability to pair them together. Nevertheless, because we explicitly directed our participants to actively attend to both items of a pair and associate them together (unlike in the passive viewing of scenes in the emotional trade-off task), we thought it more likely that negative face-object pairs, having been integrated into a representational unit, would be significantly better remembered than neutral pairs, particularly in the nap group. It is possible 
that we did not find this napping benefit for the negative pairs because sleep selectively "worked on" only the salient negative objects in the pairs and not the memory for face-object pairs as a whole, similar to Bisby and Burgess (2014), suggesting that the negative pairs were not sufficiently integrated. The neutral pairs likely were able to be effectively integrated during encoding, and therefore benefitted from sleep by being better preserved compared to remaining awake. As we did not test memory for the pair components separately, we cannot say for certain that this is the case. This is a limitation of the current design, and future studies should incorporate item testing to examine the fate of the individual pair components in each valence. However, it is unlikely that this explanation fits our results as we saw (1) negative face-object pairs remembered better than neutral pairs overall, demonstrating that the ability to pair the items together was not impeded by negative salience, and (2) that sleep facilitated both negative and neutral relational memory formation. This latter point indicates that the faces paired with negative objects were just as likely to form relational connections as neutral-paired faces, which we would not expect if the sleeping brain was primarily "focusing" on consolidation of the negatively salient half of the pairs.

Alternatively, and perhaps more likely, because of the salient nature of the negative objects, the emotional face-object pairs, although encoded into an integrated memory trace to a similar degree as the neutral face-object pairs, were perhaps encoded quicker yet shallower than the neutral pairs, similar to Murray and Kensinger (2012). The findings from Murray and Kensinger (2012) support the prioritized binding theory discussed earlier, in part, but also revealed a time-dependent effect of the benefit of emotional salience. They found that when participants were asked to integrate two verbal paired associates together as one representation, given 2 seconds to encode, there was a significant mnemonic benefit for emotional-neutral pairs over neutral-neutral pairs. However, when encoding time was increased (4 or 6 seconds), the neutralneutral pair benefits increased while emotional-neutral benefits decreased. These findings indicate that mentally integrating two neutral items into an internal representation may be more time consuming but more durable than the quick and possibly more shallow encoding of emotionally salient pairs. As this idea may relate to the current findings, a deeper level of encoding might have been achieved with more elaborative processes potentially required to integrate the neutral faces with neutral objects. While the negative object-face pairs persisted in memory similarly between nap and wake groups, the neutral pairs, having a deeper memory trace, may have benefitted from sleep-based preservation and stabilization of the associations, compared to the large decay of memory in the wake group. In this case, it may be that sleep facilitation was based on the strength of encoding, as has previously been shown (Tucker \& Fishbein, 2008). Nonetheless, we acknowledge that this explanation can only go so far to explain our results, as the decay of memory for neutral pairs in the wake condition runs counter to this idea of a deeper memory trace. Future research will need to probe this theory further, perhaps by manipulating the presentation time during encoding to evaluate if this impacts subsequent preferential consolidation.

The relational pairs profited similarly from sleep, with both face-face pairs related through negative and neutral objects benefitting from the active integration and reorganization of encoded information to establish associative links between indirectly paired memory traces. As mentioned, we show that relational memory is highly correlated with direct associative memory. However, the mechanisms behind the formation and sleep-based benefit to these memories may be different for the two memory types. Because we only measured relational memory at one time point, after the retention period, it is difficult to determine when formation of the indirect associations occurred. Yet what is clear is that sleep promotes these relational memories, regardless of valence. The negative directly learned face-object pairs, having been encoded numerically better than neutral pairs at baseline, may have led to more established relationships between the A-C faces at encoding, which further benefitted from reactivation and integration of the relationships during subsequent sleep. The neutral face-face pairs may have benefitted from preferential sleep-based processes based on the deeper memory trace of the related A-B and B-C pairs, possibly with greater reactivation of these memory traces, resulting in equal performance of negative and neutral relational pairs after sleep, both significantly better than after wake.

With regard to the relationship between memory and sleep, we found a role for REM sleep in both direct associative and relational memory, albeit opposing roles. We found that REM sleep was related to more forgetting of the neutral directly paired associates over time, while it was positively related to superior neutral relational memory. These findings differ, in part, from previous literature citing a role for NREM sleep, specifically SWS, in both direct associative and relational memory. Several studies have demonstrated a relationship between aspects of SWS and memory for various forms of paired associates (Marshall, Helgadóttir, Mölle, \& Born, 2006; Tucker \& Fishbein, 2008; Studte, Bridger, \& Mecklinger, 2015). During slow wave sleep, thought to benefit hippocampal dependent declarative memory, reactivation of the face-object pairs may promote the beginning of the process of consolidation from short-term dependence on the hippocampus to eventual longterm neocortical storage (Buzsáki, 1989; Peigneux et al., 2004; Wilson \& McNaughton, 1994). Although we did not find an explicit significant relationship between performance and SWS in this study, we cannot rule out that SWS is playing a role in the preservation of memory for the paired associates, as several studies find a general benefit of sleep for this type of memory using designs that indicate a role for NREM sleep (e.g., split night design with SWS-rich sleep, Plihal \& Born, 1997; NREM 
only nap, Tucker et al., 2006; naps with and without SWS, Alger, Lau, \& Fishbein, 2012). With regard to relational memory, Lau et al. (2010), using a task similar to the current one, found a relationship between SWS and face-face memory formation. One model that could explain this relationship, at least in part, is the information overlap to abstract (iOtA) model. This model states that the reactivation of overlapping memories during SWS not only strengthen the individual memories but also serves to activate the neurons coding for the shared component (e.g., the object in the face-object pairs) even more strongly, resulting in highly potentiated interconnections between the memories (Lewis \& Durrant, 2011). However, it must be noted that while Lau et al. used a design and task similar to ours, we incorporated emotionally salient information into our stimuli, possibly leading to differently prioritized encoding of the stimuli and subsequent benefit by sleep. It is possible that the presence of both negative and neutral information in a single task creates competition and a different type of memory trace (Payne et al., 2008; Payne \& Kensinger, 2010) compared to encoding only neutral stimuli, as in Lau et al.'s study. Additionally, while both studies allowed a 90-minute nap opportunity, Lau et al. actively prevented the occurrence of REM sleep, restricting the nap to NREM only. We are unable to speculate how memory consolidation differs under these restrictions compared to our study.

We predicted a role for REM sleep in this study based in part on prior evidence showing a relationship between REM sleep and emotional memory consolidation, but also in facilitating the type of reorganization of information necessary for relational memory formation (Stickgold et al., 2000; Cai et al., 2009; Landmann et al., 2015). Indeed, we found that memory for the neutral relational face-face pairs was positively related to REM sleep. Memory for neutral direct associates, however, decayed with more REM sleep. It may be, then, that REM sleep serves not to consolidate details, such as for directly learned face-object pairs, but to foster reorganization and promote flexible use of that information. This would include forming the relational connections stemming from the directly associated pairs, and integrating both direct and indirect relationships into a new schema. Taken together, the current study provides strong evidence that sleep plays a role in both direct and indirect neutral associative memory.

\section{Summary and conclusions}

In summary, we found that a daytime nap facilitates better memory for directly learned paired associates, as well as the formation of relational associations stemming from the overlapping components of these explicitly studied pairs. The novel incorporation of emotionally negative salience into this design produced unanticipated results. Although the negative directly associated pairs were remembered better overall, possibly through prioritized binding, it was the neutral pairs that were preferentially preserved with a nap. Although not conclusive, it may be that the neutral pairs, being more difficult to bind together, create a stronger connection between the item representations and a deeper level of encoding, which may have 'tagged' these pairs to benefit from preferential consolidation during sleep. Relational memory, which is dependent on how well the direct associations are learned in the first place, also benefitted from sleep, and this was true for both neutral and negative relational pairs. This likely occurred through connections forged between indirectly related faces during wake and optimally integrated during subsequent sleep. Although there was no relationship between SWS and either direct or relational memory, we did find that REM sleep was related to a greater decline in memory for direct associations over time. But interestingly, memory for relational associations increased with REM sleep, a time during which intracortical communication allows the neocortical representations of the direct pairs to reorganize to establish relational connections.

It should be noted that these results were obtained after only a relatively brief period of daytime sleep, consisting of about 60 minutes of total sleep time, on average. We point this out for two reasons. First, if a nap has the ability to produce significant differences in performance, it is quite possible that a longer duration of sleep, such as overnight sleep, could further benefit and differentiate the contribution of emotional salience to both direct associative and relational memory. Future studies that involve longer durations of sleep should examine such effects. Second, the importance of sleep in not only veridical memory consolidation but also in promoting the type of flexible application of memory we see with relational memory formation is apparent here. The tendency for sleep to be one of the first things sacrificed in one's daily life to increase productivity is a destructive habit. As evidenced by this study, even a brief daytime nap can help preserve information and promote reorganization of this information to boost its subsequent adaptive flexible use.

Acknowledgements The authors would like to thank Michael Dai, Tony Cunningham, and Enma Pardilla-Delgado for their constructive contribution to this manuscript.

\section{References}

Alger, S. E., Lau, H., \& Fishbein, W. (2012). Slow wave sleep during a daytime nap is necessary for protection from subsequent interference and long-term retention. Neurobiology of Learning and Memory, 98(2), 188-196.

Baran, B., Pace-Schott, E. F., Ericson, C., \& Spencer, R. M. (2012). Processing of emotional reactivity and emotional memory over sleep. The Journal of Neuroscience, 32(3), 1035-1042. 
Beck, A. T., Epstein, N., Brown, G., \& Steer, R. A. (1988). An inventory for measuring clinical anxiety: Psychometric properties. Journal of Consulting and Clinical Psychology, 56(6), 893.

Beck, A. T., Ward, C., \& Mendelson, M. (1961). Beck depression inventory (BDI). Archives of General Psychiatry, 4(6), 561-571.

Bennion, K. A., Mickley Steinmetz, K. R., Kensinger, E. A., \& Payne, J. D. (2015). Sleep and cortisol interact to support memory consolidation. Cerebral Cortex, 25(3), 646-657. doi: 10.1093/cercor/bht255

Bisby, J. A., \& Burgess, N. (2014). Negative affect impairs associative memory but not item memory. Learning \& Memory, 21(1), 760-766.

Bunsey, M., \& Eichenbaum, H. (1996). Conservation of hippocampal memory function in rats and humans. Nature, 379(6562), 255-257.

Buysse, D. J., Reynolds, C. F., III, Monk, T. H., Berman, S. R., \& Kupfer, D. J. (1989). The Pittsburgh sleep quality index: A new instrument for psychiatric practice and research. Psychiatry Research, 28(2), 193-213.

Buzsáki, G. (1989). Two-stage model of memory trace formation: A role for "noisy" brain states. Neuroscience, 31(3), 551-570.

Cai, D. J., Mednick, S. A., Harrison, E. M., Kanady, J. C., \& Mednick, S. C. (2009). REM, not incubation, improves creativity by priming associative networks. Proceedings of the National Academy of Sciences of the United States of America, 106(25), 10130-10134.

Cunningham, T. J., Crowell, C. R., Alger, S. E., Kensinger, E. A., Villano, M. A., Mattingly, S. M., \& Payne, J. D. (2014). Psychophysiological arousal at encoding leads to reduced reactivity but enhanced emotional memory following sleep. Neurobiology of Learning and Memory, 114, 155-164.

Davachi, L., \& Wagner, A. D. (2002). Hippocampal contributions to episodic encoding: Insights from relational and item-based learning. Journal of Neurophysiology, 88(2), 982-990.

Durrant, S. J., Cairney, S. A., McDermott, C., \& Lewis, P. A. (2015). Schema-conformant memories are preferentially consolidated during REM sleep. Neurobiology of Learning and Memory, 122, 41-50.

Dusek, J. A., \& Eichenbaum, H. (1997). The hippocampus and memory for orderly stimulus relations. Proceedings of the National Academy of Sciences of the United States of America, 94(13), 7109-7114.

Eichenbaum, H. (2004). Hippocampus: Cognitive processes and neural representations that underlie declarative memory. Neuron, 44(1), $109-120$.

Eichenbaum, H., \& Cohen, N. J. (2001). From conditioning to conscious recollection: Memory systems of the brain. New York, NY: Oxford University Press.

Ellenbogen, J. M., Hu, P. T., Payne, J. D., Titone, D., \& Walker, M. P. (2007). Human relational memory requires time and sleep. Proceedings of the National Academy of Sciences of the United States of America, 104(18), 7723-7728.

Gais, S., \& Born, J. (2004). Declarative memory consolidation: Mechanisms acting during human sleep. Learning \& Memory, 11(6), 679-685.

Hadley, C. B., \& MacKay, D. G. (2006). Does emotion help or hinder immediate memory? Arousal versus priority-binding mechanisms. Journal of Experimental Psychology: Learning, Memory, and Cognition, 32(1), 79.

Hasselmo, M. E., \& Bower, J. M. (1993). Acetylcholine and memory. Trends in Neurosciences, 16(6), 218-222.

Hoddes, E., Zarcone, V., Smythe, H., Phillips, R., \& Dement, W. (1973). Quantification of sleepiness: A new approach. Psychophysiology, 10(4), 431-436.

Horne, J. A., \& Ostberg, O. (1976). A self-assessment questionnaire to determine morningness-eveningness in human circadian rhythms. International Journal of Chronobiology, 4(2), 97-110.

Hu, P., Stylos-Allan, M., \& Walker, M. P. (2006). Sleep facilitates consolidation of emotional declarative memory. Psychological Science, 17(10), 891-898.

Kensinger, E. A. (2009). What factors need to be considered to understand emotional memories? Emotion Review, 1(2), 120-121.
Kensinger, E. A., Garoff-Eaton, R. J., \& Schacter, D. L. (2006). Memory for specific visual details can be enhanced by negative arousing content. Journal of Memory and Language, 54(1), 99-112.

Landmann, N., Kuhn, M., Maier, J. G., Spiegelhalder, K., Baglioni, C., Frase, L., ... Nissen, C. (2015). REM sleep and memory reorganization: Potential relevance for psychiatry and psychotherapy. Neurobiology of Learning and Memory, 122, 28-40.

Lau, H., Alger, S. E., \& Fishbein, W. (2011). Relational memory: A daytime nap facilitates the abstraction of general concepts. PLoS ONE, 6(11), e27139.

Lau, H., Tucker, M., \& Fishbein, W. (2010). Daytime napping: Effects on human direct associative and relational memory. Neurobiology of Learning and Memory, 93(4), 554-560.

Lewis, P. A., \& Durrant, S. J. (2011). Overlapping memory replay during sleep builds cognitive schemata. Trends in Cognitive Sciences, 15(8), 343-351.

Loftus, E. F., Loftus, G. R., \& Messo, J. (1987). Some facts about "weapon focus". Law and Human Behavior, 11(1), 55.

MacKay, D. G., \& Ahmetzanov, M. V. (2005). Emotion, memory, and attention in the taboo Stroop paradigm. Psychological Science, 16(1), 25-32.

MacKay, D. G., Shafto, M., Taylor, J. K., Marian, D. E., Abrams, L., \& Dyer, J. R. (2004). Relations between emotion, memory, and attention: Evidence from taboo Stroop, lexical decision, and immediate memory tasks. Memory \& Cognition, 32(3), 474-488.

Marshall, L., Helgadóttir, H., Mölle, M., \& Born, J. (2006). Boosting slow oscillations during sleep potentiates memory. Nature, 444(7119), 610-613.

Mather, M., \& Sutherland, M. R. (2011). Arousal-biased competition in perception and memory. Perspectives on Psychological Science, 6(2), 114-133.

Murray, B. D., \& Kensinger, E. A. (2012). The effects of emotion and encoding strategy on associative memory. Memory \& Cognition, 40(7), 1056-1069.

Nishida, M., Pearsall, J., Buckner, R. L., \& Walker, M. P. (2009). REM sleep, prefrontal theta, and the consolidation of human emotional memory. Cerebral Cortex, 19(5), 1158-1166.

Nummenmaa, L., Hyönä, J., \& Calvo, M. G. (2006). Eye movement assessment of selective attentional capture by emotional pictures. Emotion, 6(2), 257.

Pavlides, C., \& Winson, J. (1989). Influences of hippocampal place cell firing in the awake state on the activity of these cells during subsequent sleep episodes. The Journal of Neuroscience: The Official Journal of the Society for Neuroscience, 9(8), 2907-2918.

Payne, J. D., Chambers, A. M., \& Kensinger, E. A. (2012). Sleep promotes lasting changes in selective memory for emotional scenes. Frontiers in Integrative Neuroscience, 6. doi:10.3389 /fnint.2012.00108

Payne, J. D., \& Kensinger, E. A. (2010). Sleep's role in the consolidation of emotional episodic memories. Current Directions in Psychological Science, 19(5), 290-295.

Payne, J. D., \& Kensinger, E. A. (2011). Sleep leads to changes in the emotional memory trace: Evidence from FMRI. Journal of Cognitive Neuroscience, 23(6), 1285-1297.

Payne, J. D., Kensinger, E. A., Wamsley, E. J., Spreng, R. N., Alger, S. E., Gibler, K., ... Stickgold, R. (2015). Napping and the selective consolidation of negative aspects of scenes. Emotion, 15(2), 176.

Payne, J. D., Stickgold, R., Swanberg, K., \& Kensinger, E. A. (2008). Sleep preferentially enhances memory for emotional components of scenes. Psychological Science, 19(8), 781-788.

Peigneux, P., Laureys, S., Fuchs, S., Collette, F., Perrin, F., Reggers, J., . . Maquet, P. (2004). Are spatial memories strengthened in the human hippocampus during slow wave sleep? Neuron, 44(3), 535-545.

Phelps, E. A., Ling, S., \& Carrasco, M. (2006). Emotion facilitates perception and potentiates the perceptual benefits of attention. Psychological Science, 17(4), 292-299. 
Plihal, W., \& Born, J. (1997). Effects of early and late nocturnal sleep on declarative and procedural memory. Journal of Cognitive Neuroscience, 9(4), 534-547.

Preston, A. R., \& Eichenbaum, H. (2013). Interplay of hippocampus and prefrontal cortex in memory. Current Biology, 23(17), R764-R773.

Preston, A. R., Shrager, Y., Dudukovic, N. M., \& Gabrieli, J. D. (2004). Hippocampal contribution to the novel use of relational information in declarative memory. Hippocampus, 14(2), 148-152.

Rechtschaffen, A., \& Kales, A. (1968). A manual standardized terminology, techniques and scoring system for sleep stages of human subjects. Bethesda, Maryland, USA: U.S. Department of Health.

Sirota, A., \& Buzsáki, G. (2005). Interaction between neocortical and hippocampal networks via slow oscillations. Thalamus \& Related Systems, 3(04), 245-259.

Spielberger, C. D. (2010). State-trait anxiety inventory. In I. B. Weiner, \& W. E. Craighead (Eds.), Corsini encyclopedia of psychology (4th ed.). New York, NY: Wiley.

Staresina, B. P., Alink, A., Kriegeskorte, N., \& Henson, R. N. (2013). Awake reactivation predicts memory in humans. Proceedings of the National Academy of Sciences, 110(52), 21159-21164.

Studte, S., Bridger, E., \& Mecklinger, A. (2015). Nap sleep preserves associative but not item memory performance. Neurobiology of Learning and Memory, 120, 84-93.

Sutherland, G. R., \& McNaughton, B. (2000). Memory trace reactivation in hippocampal and neocortical neuronal ensembles. Current Opinion in Neurobiology, 10(2), 180-186.

Tambini, A., Ketz, N., \& Davachi, L. (2010). Enhanced brain correlations during rest are related to memory for recent experiences. Neuron, 65(2), 280-290.

Tamminen, J., Payne, J. D., Stickgold, R., Wamsley, E. J., \& Gaskell, M. G. (2010). Sleep spindle activity is associated with the integration of new memories and existing knowledge. The Journal of
Neuroscience: The Official Journal of the Society for Neuroscience, 30(43), 14356-14360.

Thomaz, C. E., \& Giraldi, G. A. (2010). A new ranking method for principal components analysis and its application to face image analysis. Image and Vision Computing, 28(6), 902-913.

Tucker, M. A., \& Fishbein, W. (2008). Enhancement of declarative memory performance following a daytime nap is contingent on strength of initial task acquisition. Sleep, 31(2), 197-203.

Tucker, M. A., Hirota, Y., Wamsley, E. J., Lau, H., Chaklader, A., \& Fishbein, W. (2006). A daytime nap containing solely non-REM sleep enhances declarative but not procedural memory. Neurobiology of Learning and Memory, 86(2), 241-247.

van Kesteren, M. T., Fernández, G., Norris, D. G., \& Hermans, E. J. (2010). Persistent schema-dependent hippocampal-neocortical connectivity during memory encoding and postencoding rest in humans. Proceedings of the National Academy of Sciences, 107(16), 7550-7555.

Wagner, U., Gais, S., \& Born, J. (2001). Emotional memory formation is enhanced across sleep intervals with high amounts of rapid eye movement sleep. Learning \& Memory, 8(2), 112-119.

Walker, M. P., Liston, C., Hobson, J. A., \& Stickgold, R. (2002). Cognitive flexibility across the sleep-wake cycle: REM-sleep enhancement of anagram problem solving. Cognitive Brain Research, 14(3), 317-324.

Watson, D., Clark, L. A., \& Tellegen, A. (1988). Development and validation of brief measures of positive and negative affect: The PANAS scales. Journal of Personality and Social Psychology, 54(6), 1063.

Zeithamova, D., Dominick, A. L., \& Preston, A. R. (2012). Hippocampal and ventral medial prefrontal activation during retrieval-mediated learning supports novel inference. Neuron, 75(1), 168-179. 